\title{
Isthmin exerts pro-survival and death-promoting effect on endothelial cells through alphavbeta5 integrin depending on its physical state
}

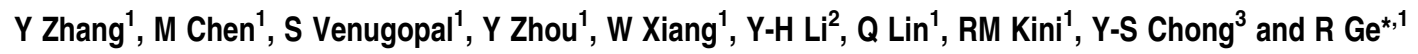

Isthmin (ISM) is a $60 \mathrm{kDa}$ secreted-angiogenesis inhibitor that suppresses tumor growth in mouse and disrupts vessel patterning in zebrafish embryos. It selectively binds to alphavbeta5 $(\alpha \mathrm{v} \beta 5)$ integrin on the surface of endothelial cells (ECs), but the mechanism of its antiangiogenic action remains unknown. In this work, we establish that soluble ISM suppresses in vitro angiogenesis and induces EC apoptosis by interacting with its cell surface receptor $\alpha \mathrm{V} \beta 5$ integrin through a novel 'RKD' motif localized within its adhesion-associated domain in MUC4 and other proteins domain. ISM induces EC apoptosis through integrinmediated death (IMD) by direct recruitment and activation of caspase-8 without causing anoikis. On the other hand, immobilized ISM loses its antiangiogenic function and instead promotes EC adhesion, survival and migration through $\alpha \mathrm{v} \beta 5$ integrin by activating focal adhesion kinase (FAK). ISM unexpectedly has both a pro-survival and death-promoting effect on ECs depending on its physical state. This dual function of a single antiangiogenic protein may impact its antiangiogenic efficacy in vivo.

Cell Death and Disease (2011) 2, e153; doi:10.1038/cddis.2011.37; published online 5 May 2011

Subject Category: Experimental medicine

Angiogenesis, formation of new blood vessels from preexisting capillaries, is critical for embryonic development, wound healing, reproduction, as well as tumor progression and metastasis. ${ }^{1}$ Angiogenesis inhibitors hence offer new promises as therapeutic agents for cancer therapy. In vivo, angiogenesis is believed to be regulated by a balance of pro and antiangiogenic factors. Although a number of endogenous angiogenesis inhibitors such as thrombospondin-1 (TSP-1) and endostatin have been discovered, the full spectrum of angiogenesis inhibitors in vivo and how they regulate angiogenesis remain to be fully elucidated. ${ }^{2}$

Isthmin (ISM), a $60 \mathrm{kDa}$ secreted matricellular protein, was originally identified as a gene highly expressed in the midbrain-hindbrain organizer in Xenopus embryos. ${ }^{3}$ It contains a centrally located thrombospondin type 1 repeat (TSR) domain and a C-terminal domain called adhesion-associated domain in MUC4 and other proteins (AMOP). We recently reported that ISM is a novel endogenous angiogenesis inhibitor with functions in both physiological and pathological angiogenesis. ${ }^{4}$ It suppresses multiple aspects of in vitro angiogenesis including endothelial cell (EC) capillary network formation on Matrigel and EC proliferation. It also induces EC apoptosis. In mice, overexpression of ISM in B16 melanoma cells inhibited tumor growth and angiogenesis. Knockdown of ISM in zebrafish embryos led to abnormal intersegmental vessel (ISV) formation in the trunk. ${ }^{4}$ Although ISM was shown to selectively bind to alphavbeta5 $(\alpha \mathrm{v} \beta 5)$ integrin, the mechanism of its antiangiogenic action is still largely unclear.

To decipher ISM's mechanism of action, we addressed the following questions in this work: (1) Does $\alpha v \beta 5$ integrin mediate ISM's antiangiogenic activities, such as inducing apoptosis? (2) How does ISM induce EC apoptosis? (3) Would ISM generate similar anti-endothelial effects in both soluble and anchored conditions? (4) Would ISM function as a cell adhesion molecule? If yes, how does it compare with other cell adhesion molecule in the extracellular matrix (ECM)? Using both neutralizing antibody as well as RNAi gene expression knockdown, we demonstrated that suppressing integrin $\alpha v \beta 5$ could stifle apoptosis induced by ISM and relieve ISM's inhibition of EC proliferation. We further established that ISM induces EC apoptosis through integrinmediated death (IMD) by activating caspase-8 without causing anoikis. Although soluble ISM potently induces apoptosis, immobilized ISM loses the proapoptotic activity and instead promotes EC adhesion, survival and haptotactic migration. These results suggest that the matricellular protein ISM in different physical state can trigger different intracellular signaling pathways and generate different biological effects through the same integrin receptor. It can function as an integrin agonist as well as an antagonist. This is different from classical ECM integrin ligands such as vitronectin (VN) or fibronectin (FN).

\footnotetext{
${ }^{1}$ Department of Biological Sciences, National University of Singapore, Block S2, Science Drive 4, Singapore 117543, Singapore; ${ }^{2}$ Institute of Medical Biology, A*STAR, 8A Biomedical Grove, Immunos, Singapore 138648, Singapore and ${ }^{3}$ Department of Obstetrics and Gynaecology, Yong Loo Lin School of Medicine, National University of Singapore, Singapore 119074, Singapore

${ }^{*}$ Corresponding author: R Ge, Department of Biological Sciences, National University of Singapore, Block S2, 05-04, Science drive 4, Singapore 117543, Singapore. Tel: + 65 65167879; Fax: + 65 67792486; E-mail: dbsgerw@ nus.edu.sg

Keywords: isthmin; angiogenesis; integrin-mediated death; apoptosis; physical state

Abbreviations: ISM, isthmin; EC, endothelial cells; IMD, integrin mediated death; FAK, focal adhesion kinase; TSP-1, thrombospondin-1; TSR, thrombospondin type 1 repeat; AMOP, adhesion-associated domain in MUC4 and other proteins; ISV, intersegmental vessel; ECM, extracellular matrix; VEGF, vascular endothelial growth factor; HUVEC, human umbilical vein endothelial cells; FADD, fas-associated protein with death domain; VN, vitronectin; FN, fibronectin

Received 19.11.10; revised 21.3.11; accepted 29.3.11; Edited by P Salomoni
} 


\section{Results}

Soluble ISM inhibits angiogenesis and induces EC apoptosis through $\alpha \mathbf{v} \beta 5$ integrin. We have previously reported that soluble ISM produced from $E$. coli functions as an angiogenesis inhibitor, inhibiting EC tube formation, inducing EC apoptosis and suppressing vascular endothelial growth factor (VEGF)-stimulated EC proliferation in a dosedependent manner. ${ }^{4}$ We also found that ISM selectively binds to $\alpha v \beta 5$, but not other integrins expressed in ECs. ${ }^{4} \mathrm{We}$ show here that recombinant ISM protein produced from cultured mammalian cells (HEK293T) has similar antiangiogenic and apoptosis-induction activity (Supplementary Figure S1). Because of cost-effective production, recombinant ISM made from bacteria is used in this study.

As $\alpha v \beta 5$ integrin is a cell surface receptor of ISM, we wish to determine whether ISM inhibits angiogenesis and induces apoptosis through this integrin. In ECs (human umbilical vein endothelial cells, HUVEC) used in this study, integrin $\beta 3$ or $\beta 5$ subunit only pairs with $\alpha \mathrm{v}$ subunit, with $\alpha \mathrm{v} \beta 3$ expressed at a much higher level than $\alpha v \beta 5 .{ }^{5,6}$ Knockdown of $\beta 3$ or $\beta 5$ integrin subunit expression in ECs had no effect on cell adhesion on gelatin, FN or collagen I (data not shown), possibly due to the presence of $\beta 1$ integrins such as $\alpha 5 \beta 1$ in these cells. It is known that $\beta 1$ integrin mediate cell adhesion to gelatin, FN and collagen I. ${ }^{7}$ Hence the effect of knockdown of $\beta 3$ or $\beta 5$ integrin subunit expression on ISM-mediated inhibition of EC tube formation and induction of apoptosis was carried out in cells seeded on gelatin. As shown in Figure 1a, introduction of siRNA significantly reduced $\beta 3$ and $\beta 5$-integrin expression up to $70 \%$ in ECs. However, only knockdown of $\beta 5$-integrin expression alleviated ISM-mediated inhibition of tube formation (Figure 1b). Similarly, ISM-induced EC apoptosis was selectively suppressed by $\beta 5$, but not $\beta 3$ knockdown (Figure 2a). In the absence of ISM, knockdown of these integrins did not induce apoptosis. Similarly, only knockdown of $\beta 5$-integrin expression significantly rescued ISM-mediated inhibition of VEGF-induced EC proliferation (Figure $2 b$ ). These results demonstrate that $\alpha v \beta 5$ integrin has an essential role in ISM-mediated angiogenesis inhibition and apoptosis.

As $\alpha v \beta 5$ is essential for ISM-induced apoptosis, the amount of free $\alpha \mathrm{v} \beta 5$ available for ISM binding should correspond to the extent of EC apoptosis. In ECs attached on VN- or ISM-coated surface, both ligands of $\alpha v \beta 5$ integrin, the free $\alpha v \beta 5$ available for soluble ISM binding are reduced, comparing with ECs attached on FN-coated surface (Figure 2c), most likely due to $\alpha v \beta 5$ occupancy by VN or ISM. Correspondingly, soluble ISM induced only marginal apoptosis in ECs attached on VN and ISM, compared with significant apoptosis with ECs attached on FN (Figure 2d).

Together, these results demonstrate that soluble ISM suppresses angiogenesis and induces EC apoptosis through interacting with $\alpha \mathrm{v} \beta 5$ integrin on EC surface without causing anoikis. The extent of ISM-induced apoptosis depends on the number of free $\alpha \mathrm{v} \beta 5$ integrin on EC surface that are available for soluble ISM binding.

Soluble ISM induces EC apoptosis through IMD. It is known that certain cyclic RGD peptides ${ }^{8}$ or non-peptide RGD mimetics ${ }^{9}$ induce EC detachment from adhered surface (de-adhesion) through binding to integrins, and hence causing apoptosis through anoikis. To determine whether ISM induces EC apoptosis through anoikis, we examined whether ISM induces detachment of adherent ECs. The adhesion of ECs on different immobilized ECM proteins is mediated by distinct integrins. For example, EC adhesion to $\mathrm{VN}$ is mediated primarily by $\alpha \mathrm{v} \beta 3$ and $\alpha \mathrm{v} \beta 5$ integrins, whereas EC adhesion to FN occurred mainly through $\alpha 5 \beta 1$ integrin. ${ }^{5}$ As shown in supplementary (Supplementary Figure S2), ISM added into the culture media did not induce
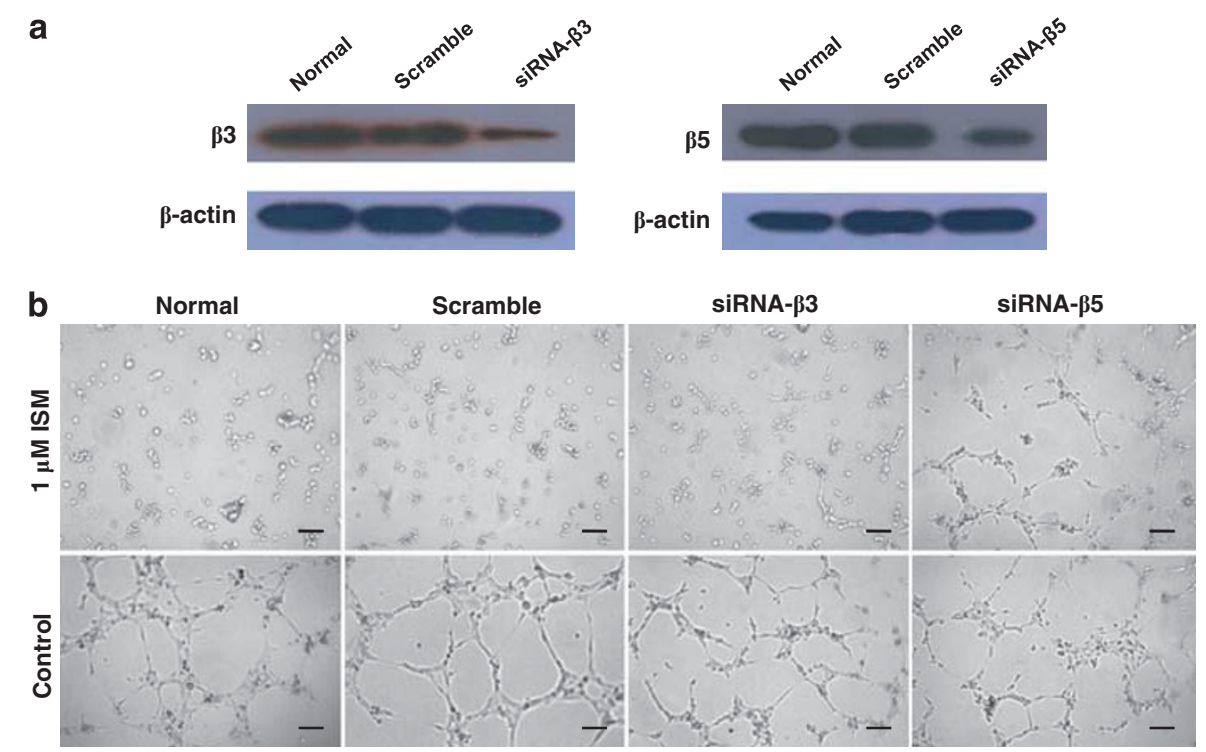

Figure 1 ISM inhibits in vitro angiogenesis through $\alpha \mathrm{v} \beta 5$ integrin. (a) Transient transfection of $\beta 3$ and $\beta 5$ siRNA effectively knocked down their expression in ECs. Same amount of corresponding scrambled siRNA are transfected as control. (b) Only knockdown of $\beta 5$-integrin expression abolished the anti-tube formation activity of ISM 

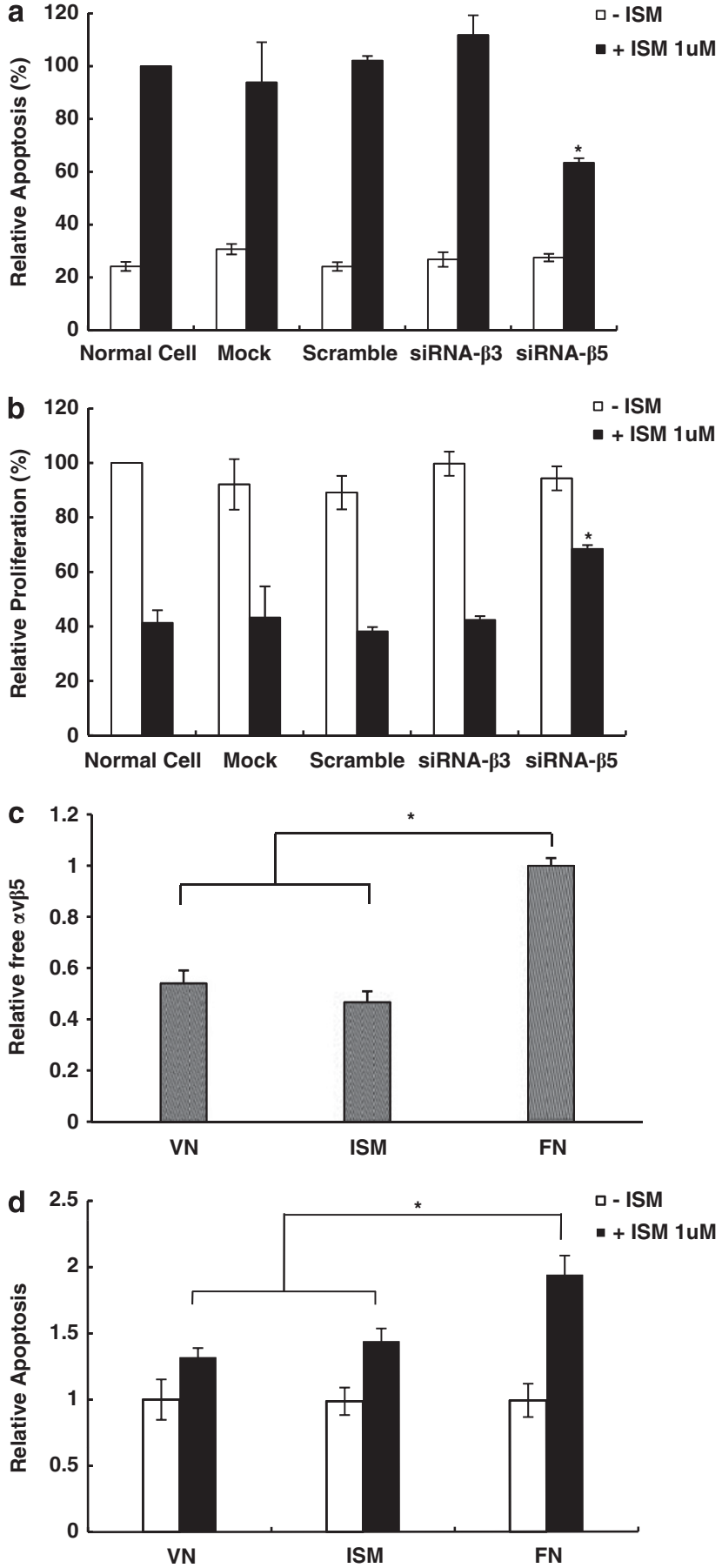

Figure 2 ISM induces EC apoptosis through $\alpha \mathrm{v} \beta 5$ integrin. (a) Knockdown of $\beta 5$, but not $\beta 3$ integrin, suppressed ISM-induced apoptosis. (b) Knockdown of $\beta 5$ integrin rescued ISM-suppressed proliferation. (c) More free $\alpha \mathrm{v} \beta 5$ integrins are available in ECs growing on FN-coated surface. VN, vitronectin; ISM, isthmin; FN, fibronectin. (d) ISM induces more extensive apoptosis in ECs grown on FN-coated surface

de-adhesion of ECs plated on FN- or VN-coated surface, regardless of whether its receptor $\alpha \mathrm{v} \beta 5$ integrin is bound by a ligand (VN) or not (FN). Under the same condition, ISM potently induced EC apoptosis (Xiang et al. ${ }^{4}$ and Figure 2a).

We previously established that ISM induces EC apoptosis through a caspase-dependent pathway. ${ }^{4}$ To further decipher the mechanism of ISM-induced apoptosis, we used specific inhibitors for caspase- $3,-8$ and -9 to block the extrinsic and intrinsic apoptosis pathways. As shown in Figure 3a, caspase-3 and -8 inhibitors effectively prevented ISM-induced apoptosis, whereas caspase-9-specific inhibitor had no effect.

A gradual activation of caspase- 8 and caspase- 3 under ISM treatment was observed (Figure $3 \mathrm{~b}$ ). The active form of caspase-8 $(20 \mathrm{kDa})$ was first detected at $4 \mathrm{~h}$ post ISM treatment, whereas activated caspase-3 was first detected after $16 \mathrm{~h}$ ISM treatment. No cleaved form of caspase- 9 was detected even after $24 \mathrm{~h}$ treatment. As a hallmark indicator of apoptosis, the cleaved form of poly ADP ribose polymerase (PARP, $87 \mathrm{kDa}$ ) was also found to gradually increase (Figure 3b). Activation of caspase- 8 and caspase-3 by ISM depends on $\alpha \mathrm{v} \beta 5$ integrin as knockdown of $\beta 5$ integrin by siRNA-suppressed ISM-induced caspase activations and PARP cleavage (Figure 3c). These results established that ISM induces apoptosis via the extrinsic apoptosis pathway through caspase-8 activation.

It has been previously reported that unligated or antagonized integrin can cluster on cell surface and subsequently recruit caspase- 8 to plasma membrane, where it becomes activated in a death receptor-independent manner, a phenomenon termed IMD. ${ }^{10}$ To determine if ISM initiates apoptosis through IMD, co-immunoprecipitation was performed to examine the interaction between $\alpha v \beta 5$ integrin and caspase-8. As shown in Figure $3 d$, ISM induced the association of procaspase-8 (55 kDa) and activated caspase$8(20 \mathrm{kDa})$ with $\alpha \mathrm{v} \beta 5$ integrin. A Fas antagonist did not prevent ECs from ISM-induced apoptosis, indicating that Fas is not involved in ISM-induced cell death (Supplementary Figure S3). Correspondingly, Fas-associated protein with death domain (FADD) was not detected in ISM-induced coimmunoprecipitate sample (data not shown), indicating that FADD is not involved. We therefore conclude that soluble ISM act as a $\alpha v \beta 5$-integrin antagonist and induce EC apoptosis through IMD.

We found that soluble ISM induced integrin clustering (Figure 3E), with EC bound ISM (green) and integrin $\beta 5$-subunit (red) co-localized in cell periphery in clusters. The clustered integrin may be responsible for the recruitment and activation of caspase-8.

Immobilized ISM promotes EC survival and migration. After its secretion, ISM could potentially exist in different physical states in vivo, such as immobilized in the ECM of a tissue or soluble in circulation. Indeed, we detected ISM in the mouse plasma (98 nM) and the ECM (in Matrigel, $91.5 \mathrm{nM})$. Interestingly, when ISM was mixed with Matrigel, it lost the ability to inhibit EC tube formation. Soluble ISM at $1 \mu \mathrm{M}$ in culture medium completely abolished EC tube formation on Matrigel, whereas immobilized ISM in Matrigel had no effect on tube formation even at $3 \mu \mathrm{M}$ (Figure 4).

Integrin-mediated cell attachment on immobilized ECM ligands such as FN or VN results in cell spreading, focal adhesion and actin stress fiber formation, as well as activation of focal adhesion kinase (FAK). ${ }^{11}$ To determine if immobilized ISM could also function as a cell adhesion molecule and support EC survival, we plated ECs onto ISM-coated surface. As shown in Figure 5a, immobilized ISM supported EC 


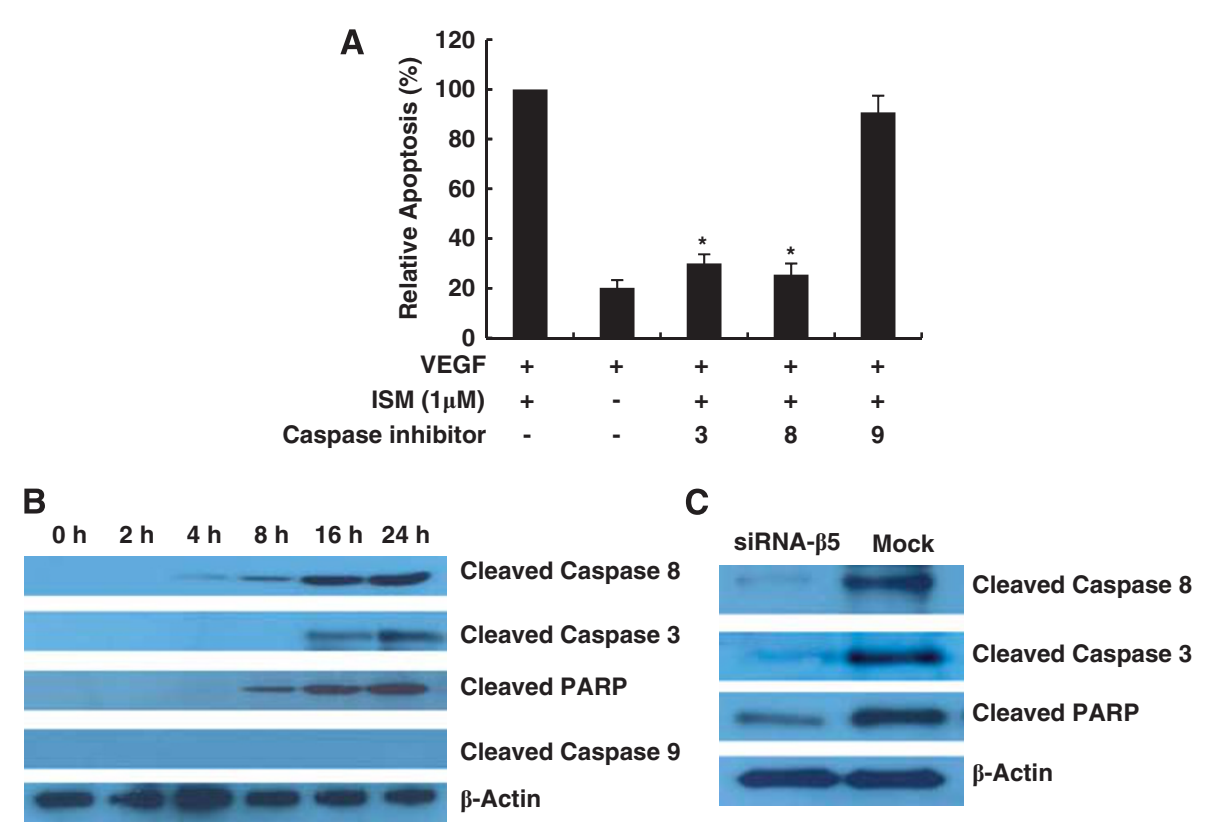

D

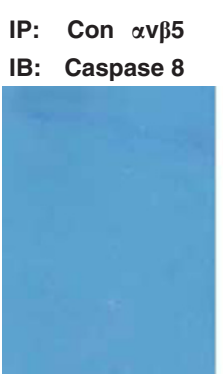

No treatment
IP: Con $\alpha \mathrm{v} \beta 5$

IB: Caspase 8

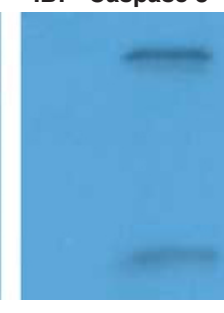

ISM treatment
Integrin- $\beta 5$

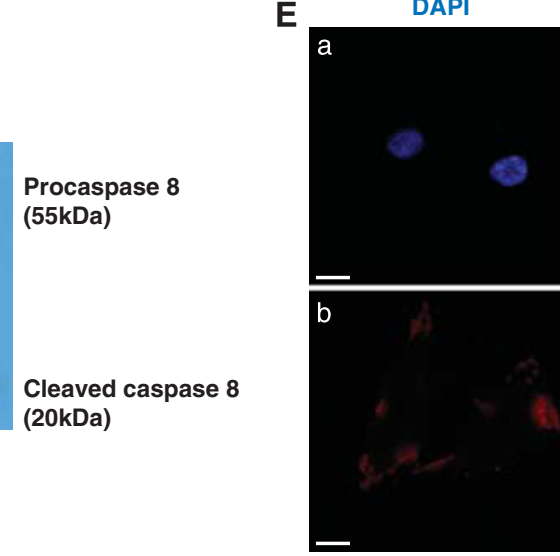

E Figure 3 ISM induces apoptosis through IMD. (A) ISM induced apoptosis through caspase-8-mediated extrinsic pathway, but not caspase-9-mediated intrinsic pathway.
(B) Kinetics of ISM-induced apoptosis. Activated caspase-8 can first be detected $4 \mathrm{~h}$ after ISM treatment. (C) Knockdown of $\beta 5$-integrin subunit by siRNA suppressed caspase-8 and caspase- 3 activation, as well as cleavage of PARP. (D) ISM induces caspase- 8 association with $\alpha \mathrm{V} \beta 5$ integrin and activation. (E) Soluble ISM induces $\alpha \mathrm{V} \beta 5$-integrin clustering. Co-localization of $\beta 5$ integrin (red) with ISM (green) is indicated by yellow signals. DAPI (blue) stains the nucleus. Scale bar: $20 \mu \mathrm{m}$

adhesion in a dose-dependent and saturable manner. ECs spread on ISM-coated surface in a similar fashion to VNcoated surface (Figure 5B, panels a and b). The adhesion and spreading was completely abolished in the presence of $10 \mathrm{mM}$ EDTA, consistent with the fact that divalent cations are essential for integrin-mediated cell adhesion ${ }^{11}$ (Figure 5B, panels $\mathrm{c}$ and $\mathrm{d}$ ). ECs attached on ISM-coated surface proliferate at similar rate, compared with ECs attached on VN or gelatin (data not shown). Immobilized ISM also induced actin stress fiber formation and integrin clustering, similar to VN (Figure 5B, panels e-h). Therefore, immobilized ISM supports EC adhesion and survival as a cell adhesion molecule.

We further demonstrated that ECs adhere to ISM-coated surface through $\alpha \mathrm{v} \beta 5$ integrin, as $\alpha \mathrm{v} \beta 5$ blocking antibody blocked $85 \%$ of this adhesion, whereas control IgG or $\alpha \mathrm{v} \beta 3$ blocking antibody had no blocking activity (Figure 5c). Anti- $\alpha \mathrm{V}$ or anti- $\beta 5$-subunit antibody also blocked EC adhesion to ISM-coated surface by 56 and $61 \%$, respectively, whereas anti- $\beta 1$ and anti- $\beta 3$ antibody had no effect. Collectively, these results confirmed that $\alpha \mathrm{v} \beta 5$ integrins mediate $\mathrm{EC}$ adhesion to ISM and serves as receptor for immobilized ISM. In comparison, EC attachment to collagen I was inhibited by anti- $\beta 1(50 \%)$, or attachment to VN was inhibited by anti- $\alpha \mathrm{v} \beta 3(51 \%)$ and anti- $\alpha \mathrm{v} \beta 5(29 \%)$, respectively, consistent with the ligand-receptor specificity (Supplementary Figure S4, $A$ and $B$ ).

Knockdown of either $\beta 3$ or $\beta 5$-integrin subunit expression by $50-70 \%$ through siRNA significantly suppressed cell adhesion on $\mathrm{VN}$-coated surface (Figure $5 \mathrm{~d}$ ), with $\beta 3$-knockdown having more potent suppression (Figure $5 \mathrm{~d}$ ). This is consistent with the much higher level of $\beta 3$ integrin expression in HUVECs 
ISM in Medium

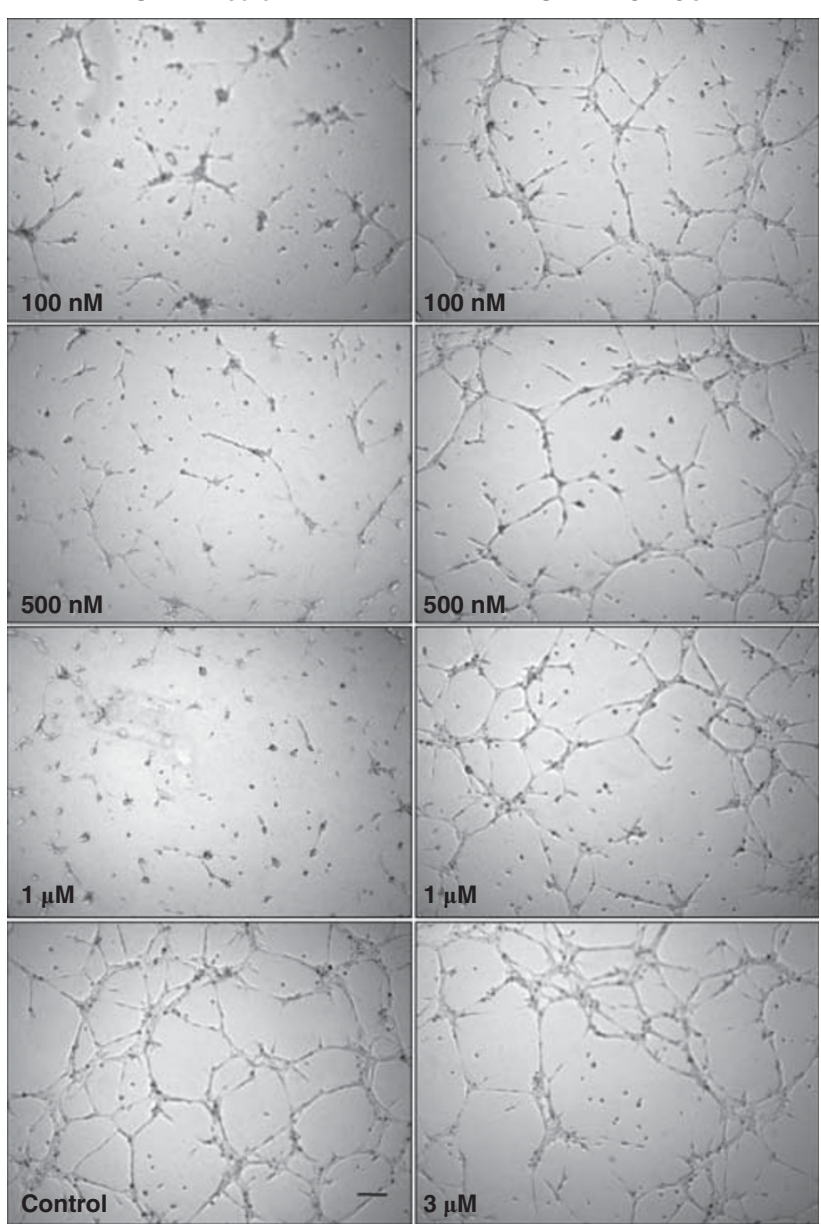

Figure 4 Immobilization of ISM by ECM abolished its anti-tube formation activity. Recombinant ISM was added into culture medium (left) or mixed into Matrigel (right). The concentrations of ISM were labeled in the panels. Scale bar represents? $\mu \mathrm{m}$. Control indicates culture medium (containing $2 \%$ FBS)

and the fact that $\alpha \mathrm{v} \beta 3$ is the main $\mathrm{VN}$ receptor. ${ }^{6,12}$ In contrast, only knockdown of $\beta 5$ integrin reduced cell adhesion to ISMcoated surface, consistent with ISM as a $\alpha \mathrm{v} \beta 5$ specific ligand (Figure $5 e$ ). Correspondingly, knockdown of $\beta 3$ or $\beta 5$ integrin subunits had no effect on EC adhesion to type I collagen, FN and gelatin (data not shown).

FAK is an important mediator of cell survival, proliferation and migration. ${ }^{13}$ Integrin clustering leads to FAK phosphorylation and subsequent recruitment to focal adhesions. ${ }^{14}$ The most crucial site for FAK activation is Y397, which is an Srcbinding site. Comparing with cells in suspension, immobilized ISM induced FAK Y397 phosphorylation, similar to immobilized VN (Figure 6a). Notably, immobilized ISM no longer induces EC apoptosis and present similar basal level apoptosis as immobilized VN, suggesting that immobilized ISM functions as an integrin agonist and transmit positive intracellular signals (Supplementary Figure S5).

ECs migrate towards a higher concentration of anchored cell adhesion molecule, a phenomenon termed haptotaxis. Immobilized ISM induced EC haptotactic migration in a concentration-dependent manner with similar potency as FN
(Figure 6b). This haptotactic migration is blocked by anti- $\alpha \mathrm{v}$, anti- $\beta 5$ and anti- $\alpha \mathrm{v} \beta 5$ antibodies, but not by anti- $\beta 1$, anti- $\beta 3$ or anti- $\alpha \mathrm{v} \beta 3$ antibodies (Figure $6 \mathrm{c}$ ), indicating $\alpha \mathrm{v} \beta 5$ as the mediator of this function.

Altogether, the above results revealed that immobilized ISM no longer induces apoptosis, but serves as an adhesion molecule for ECs and an agonist of $\alpha \mathrm{v} \beta 5$ integrin. It functions in similar fashion as $\mathrm{VN}$ or $\mathrm{FN}$, inducing $\alpha \mathrm{v} \beta 5$ integrin clustering and actin stress fiber formation, promotes EC adhesion, survival, haptotactic migration and stimulates FAK activation.

ISM binds to $\alpha v \beta 5$ integrin through a novel 'RKD' motif. Using a solid-phase ELISA-based binding assay, we determined that ISM binds $\alpha \mathrm{v} \beta 5$ integrin with a $K_{d}$ around $40 \mu \mathrm{M}$, in the range of integrin ECM ligand binding affinities (Supplementary Figure S6). We have previously determined that ISM binds to $\alpha \mathrm{v} \beta 5$ integrin through its C-terminal AMOP domain, and the AMOP domain alone can inhibit EC tube formation in vitro. ${ }^{4}$ However, there is no 'RGD' sequence within the AMOP domain of ISM. To determine how ISM binds to $\alpha \mathrm{v} \beta 5$ integrin, we produced recombinant proteins, corresponding to overlapping $\mathrm{N}$ - and $\mathrm{C}$-terminal truncations of AMOP domain (Figure 7a). ECs attached to AMOP-N coated surface with similar efficacy as the intact AMOP domain, whereas AMOP-C could not support EC attachment at all (Figure $7 b$ ). It has been reported that substitution of $G$ for $K$ in the RGD motif of a 12 residue synthetic hepatitis-A peptide did not change the physiochemical properties of the peptide. ${ }^{15}$ However, it is not known if 'RKD' can function as an integrin binding motif. In AMOP-N, there is an 'RKD' motif at amino acids 315-317. We mutated the 'KD at 316-317 position to $A A^{\prime}$ and produced the mutant $A M O P^{K D 316 A A}$ protein in the intact AMOP background (Figure 7a). As shown in Figure $7 \mathrm{~b}, \mathrm{AMOP} \mathrm{PD}^{\mathrm{K} 16 \mathrm{AA}}$ completely lost the ability to support EC adhesion. Furthermore, AMOP ${ }^{\mathrm{KD} 316 \mathrm{AA}}$ also lost the ability to inhibit EC tube formation on Matrigel (Figure 7c). These results demonstrate that ISM binds to EC surface $\alpha v \beta 5$ integrin through a novel 'RKD' motif located in its C-terminal AMOP domain. This motif has a critical role in the antiangiogenic as well as pro-survival functions of ISM. 'RKD' is therefore a novel integrin-binding motif, which binds $\alpha v \beta 5$, but not $\alpha \mathrm{v} \beta 3$ integrin.

\section{Discussion}

In this work, we demonstrated that the recently identified matricellular angiogenic inhibitor ISM functions through its cell surface receptor $\alpha \mathrm{v} \beta 5$ integrin. Although soluble ISM suppresses EC tube formation and induces EC apoptosis as an integrin antagonist, immobilized ISM functions as an integrin agonist and promotes cell survival and migration. The antiangiogenic activity of ISM is lost when ISM is immobilized in ECM. We demonstrated that mouse ISM is present in soluble (plasma) and immobilized form (extracellular matrix), thus its dual activity may have an important role in vivo.

We also identified a novel $\alpha v \beta 5$-integrin-binding site 'RKD' located in the AMOP domain of ISM. Loss of $\alpha \mathrm{V} \beta 5$ binding through point mutations of this 'RKD' motif obliterated the antiangiogenic activity of ISM in vitro and abolished the ability 

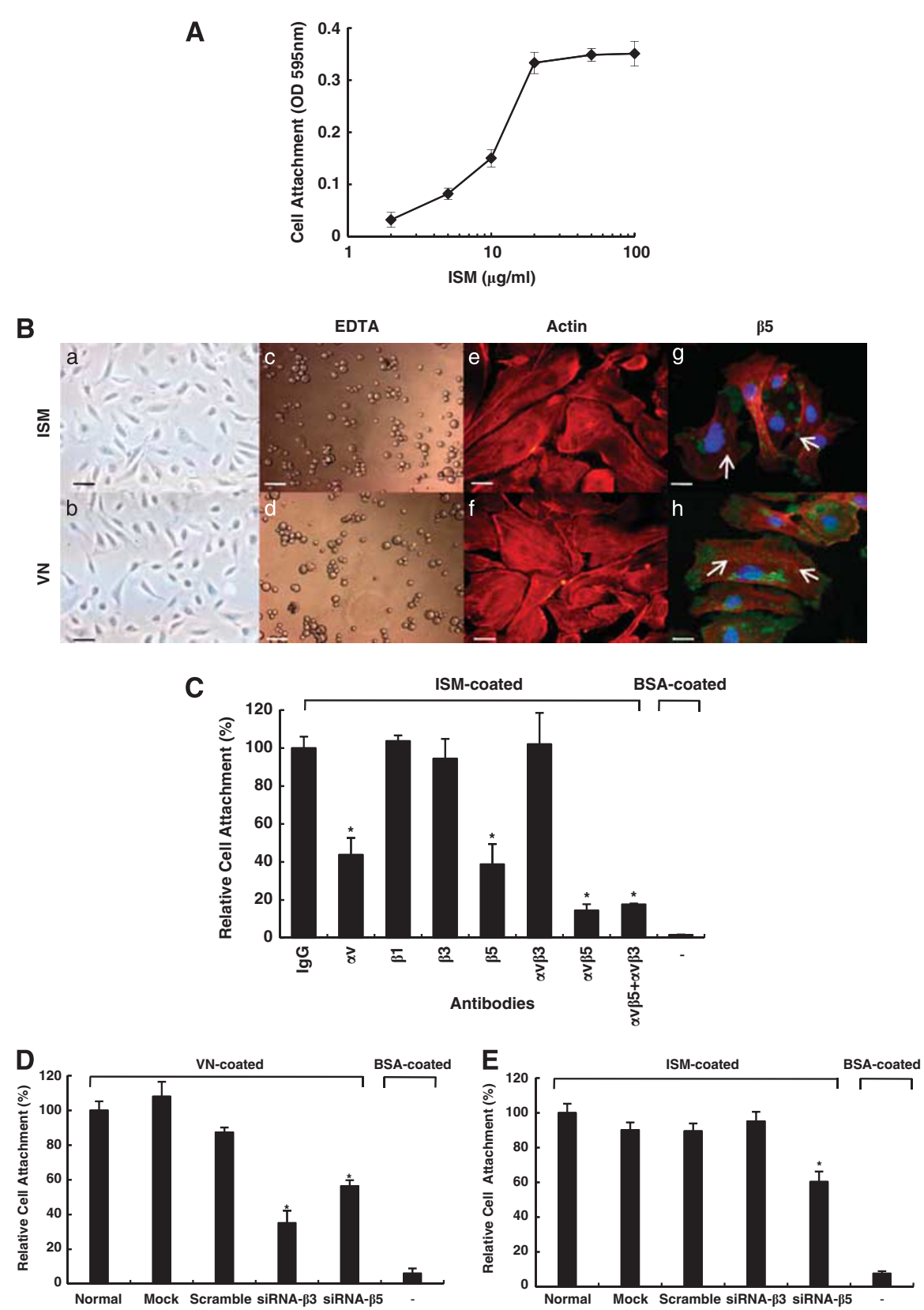

Figure 5 Immobilized ISM promotes EC adhesion through $\alpha \mathrm{v} \beta 5$ integrin. (A) ECs attach to ISM-coated surface in a dose-dependent and saturable manner. (B) Similar to VN, ISM supports EC adhesion and spreading, induces integrin clustering and actin stress fiber formation. Cell spreading in the absence or presence of EDTA are shown in phase contrast micrographs (panels A-D, scale bar: $40 \mu \mathrm{m}$ ). Actin stress fibers (red) and $\beta 5$ integrin clustering (green) are shown in panels $\mathrm{E}-\mathrm{H}$ (scale bar: $20 \mu \mathrm{m}$ ). (C) ISM mediates EC adhesion through $\alpha \mathrm{v} \beta 5$ integrin. (D) Knockdown of $\beta 3$ and $\beta 5$ integrin suppresses $\mathrm{EC}$ adhesion to VN. (E) Knockdown of $\beta 5$ integrin suppresses EC adhesion to ISM

of ISM to support EC adhesion. Therefore, integrin $\alpha \mathrm{v} \beta 5$ has an essential role in the antiangiogenic as well as pro-survival functions of ISM. This dual function of ISM through the same cell surface integrin receptor and the intracellular signaling pathways involved are summarized in Figure 8. As $\alpha \mathrm{v} \beta 5$ integrin is upregulated in tumor blood vessels, ISM most likely inhibits tumor angiogenesis through this integrin in vivo.

Similar to VN, immobilized ISM promoted EC attachment and spreading, integrin clustering, actin stress fiber formation, FAK activation and EC haptotactic migration (Figures 5 and 6). The clustering of integrins upon ECM engagement is known to transmit positional cues from ECM to intracellular signaling cascades that promote cell survival. ${ }^{14}$ Both neutralizing antibody to $\alpha v \beta 5$ integrin, as well as siRNA knockdown of $\beta 5$ integrin, abrogated EC adhesion to immobilized ISM, demonstrating that immobilized ISM is an $\alpha \mathrm{v} \beta 5$ integrin agonist (Figure 5).

Different from VN, soluble ISM inhibits in vitro angiogenesis, induces EC apoptosis and suppresses EC proliferation in an $\alpha v \beta 5$ integrin-dependent manner (Figures 1 and 2). 

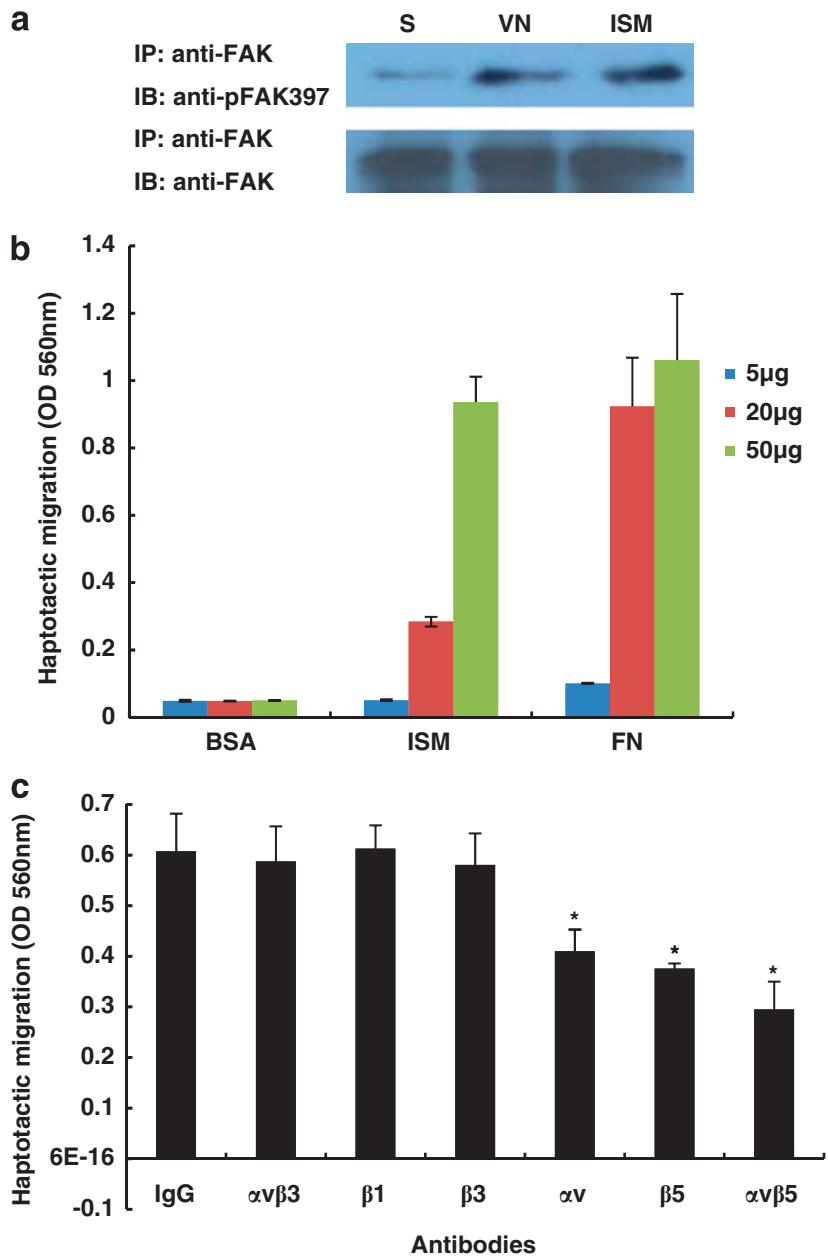

Figure 6 Immobilized ISM activates FAK and promotes EC haptotactic migration. (a) Immobilized ISM activates FAK Y397 phosphorylation in similar fashion as immobilized VN. S, cells in suspension. (b) ISM dose-dependently promotes EC haptotactic migration with similar potency as FN. (c) ISM promotes EC haptotactic migration through $\alpha \mathrm{v} \beta 5$ integrin

Knockdown of $\beta 5$, but not $\beta 3$ integrin in ECs rendered ISM inactive in inhibition of tube formation and induction of apoptosis. Correspondingly, ISM induced more extensive apoptosis in ECs cultured on non- $\alpha \mathrm{v} \beta 5$ ligand-coated surface such as FN (Figure 2d), due to the higher amount of free $\alpha \mathrm{v} \beta 5$ integrin available for soluble ISM binding in cells adhered to FN comparing with VN or ISM (Figure 2c). As ISM binds to $\alpha v \beta 5$ integrin with a low affinity $\left(K_{d}\right.$ around $\left.40 \mu \mathrm{M}\right)$, it cannot effectively displace bound $\mathrm{VN}$ by competitively binding to $\alpha \mathrm{V} \beta 5$ integrin (Supplementary Figure S6).

We further demonstrated that ISM induces apoptosis through IMD by recruiting caspase-8 to the plasma membrane and activating caspase-8 in a Fas-independent manner. Both the zymogen and active forms of caspase- 8 are found in the $\alpha \mathrm{v} \beta 5$ protein complex upon ISM induction of apoptosis (Figure 3d). Soluble ISM therefore serves as an integrin antagonist and transmits a direct death signal involving caspase- 8 and caspase- 3 . This is similar to IMDs induced by RGD containing $\alpha \mathrm{v} \beta 3$ integrin antagonist echistatin or overexpression of unligated $\alpha \mathrm{v} \beta 3$ integrins. ${ }^{10,16}$ In contrast to previous report that $\beta 5$ integrin is non-apoptotic, ${ }^{10}$ our results provide the first evidence that $\beta 5$ integrin also mediates IMD.

Both soluble and immobilized ISM induces $\alpha \mathrm{V} \beta 5$-integrin clustering (Figures $3 \mathrm{E}$ and $5 \mathrm{~B}$ ). Although clustering of unligated or antagonized integrins is known to induce apoptosis in adherent cells, clustering of agonized integrin promotes cell survival. ${ }^{17}$ Whether soluble and immobilized ISM induce different mode of integrin clustering which lead to different interactions with focal adhesion proteins and activate different intracellular signaling pathways warrant further investigation.

The full spectrum of Ism's physiological role is not clear at this moment. Our preliminary investigation indicated that $\mathrm{Ism}$ is expressed at a significantly higher level in lung, comparing with other tissues (Supplementary Figure S7). Future investigation by immunohistochemistry is needed to reveal how ISM is presented to ECs in vivo.

The phenomenon that an endogenous antiangiogenic protein in different physical state (soluble versus immobilized) carries out contrasting functions in angiogenesis may impact the in vivo efficacy of the antiangiogenic protein. This dualfunction characteristic may also exist in other endogenous antiangiogenic proteins. For example, immobilized endostatin has been reported to support EC adhesion in an integrindependent manner, whereas soluble endostatin inhibits integrin-dependent cell functions such as cell migration and induces apoptosis. ${ }^{18}$ It seems that integrin can promote or suppress apoptosis depending on the physical state of its ligand. This characteristic places integrin as a type of dependence receptors that require immobilized ligands to promote cell survival, whereas soluble ligands act as antagonists inducing IMD. ${ }^{16,19}$ In contrast to ISM, ECM adhesion molecules such as $\mathrm{VN}$ or $\mathrm{FN}$, which serve as integrin agonists when immobilized, do not function as antagonists to trigger apoptosis in soluble state.

Concluding remarks. In this work, we demonstrated that the endogenous angiogenesis inhibitor ISM could function as either antagonist or agonist of $\alpha \mathrm{v} \beta 5$ integrin and modulate EC apoptosis and survival. Although immobilized ISM promotes EC survival and haptotactic migration, soluble ISM suppresses EC tube formation and induces EC apoptosis through IMD. This study also identified a novel 'RKD' motif within the AMOP domain of ISM, which mediates the specific binding of ISM to $\alpha v \beta 5$, but not $\alpha v \beta 3$. As other endogenous angiogenesis inhibitor proteins may behave in similar fashion, it is conceivable that an antiangiogenic protein may inhibit or promote angiogenesis depending on its physical state in vivo. This may impact on the in vivo efficacy of the antiangiogenic proteins as anticancer drugs.

\section{Materials and Methods}

Cell culture and recombinant protein production. Endothelial cells (ECs) used in this work are HUVECs. Fresh umbilical cords were collected from consented maternal ward patients at the National University Hospital according to the protocol (DSRB C/00/553), which is approved by Singapore National Healthcare Group's Domain-Specific Review Board (DSRB) ethics approval committee. ECs were cultured in CSC complete medium (Cell System Corporation, Kirkland, WA, USA). Only cells of passages $4-8$ were used in experiments. 
a
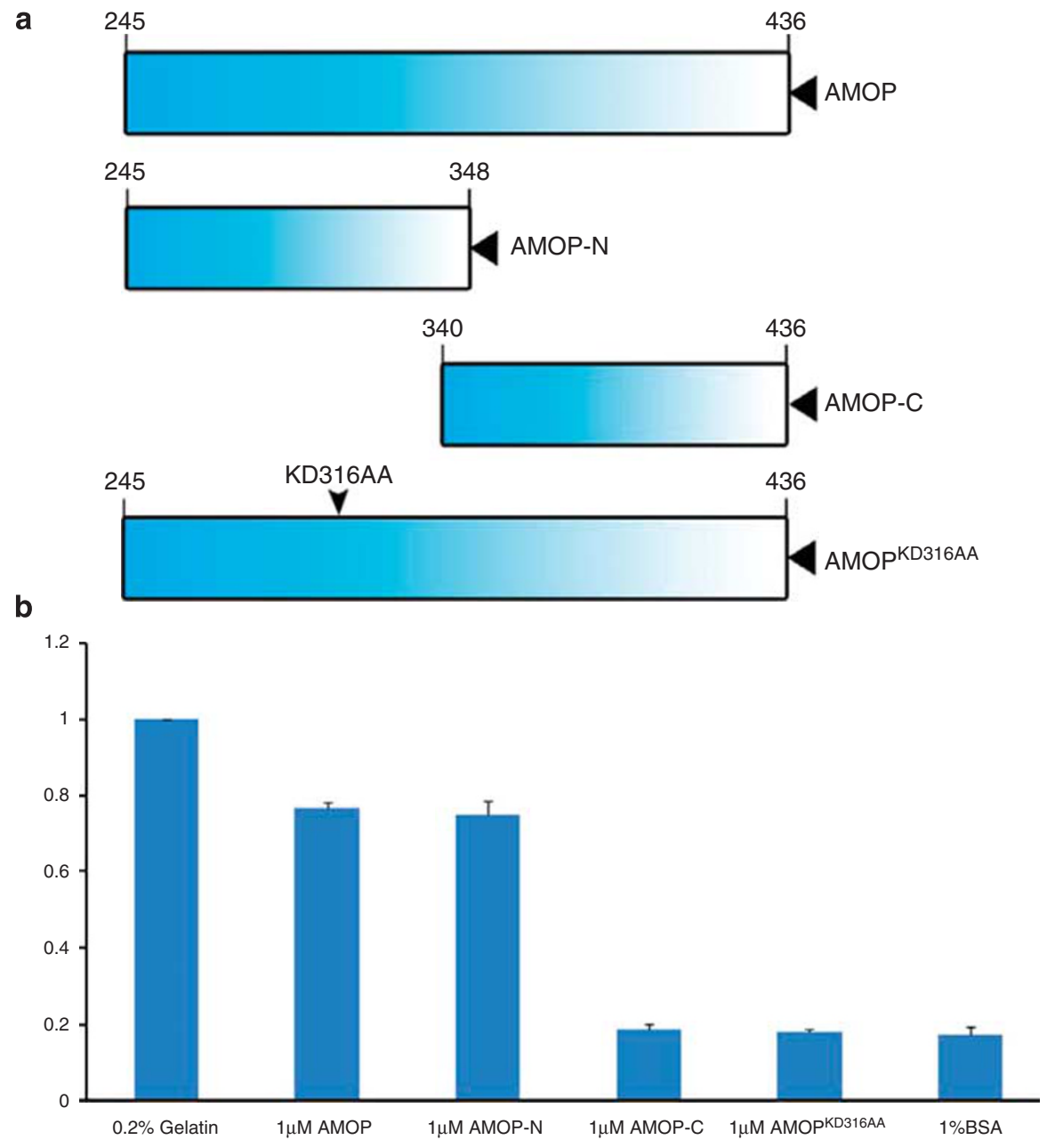

C

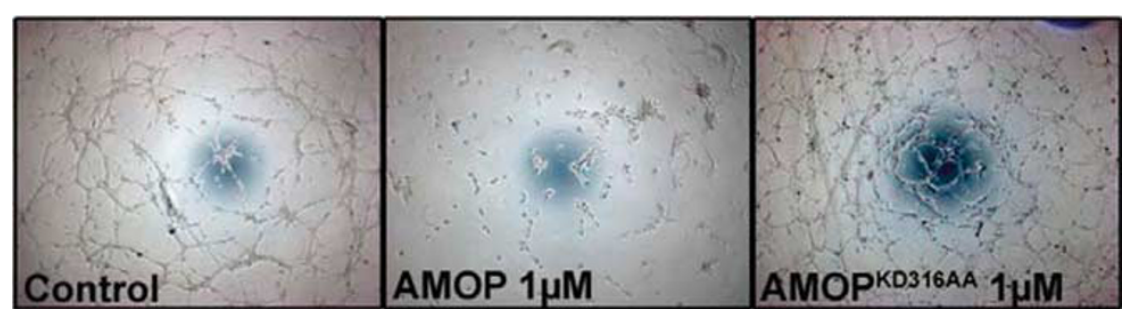

Figure 7 Identification of the $\alpha v \beta 5$ integrin-binding site in ISM. (a) Schematic diagrams showing the truncated ISM protein fragments produced as recombinant proteins in E. coli. Numbers refer to the amino acid position in the full-length secreted ISM protein. The positon of KD316AA mutation is indicated by the arrowhead. The black triangle at the right end of each truncated protein represent His-tag. (b) EC adhesion to various ISM protein fragments compared with $0.2 \%$ gelatin. $3 \%$ BSA was used as the negative control. (c) KD316AA mutation abolished the anti-tube formation activity of ISM

Cell attachment assays. The assays were performed as described previously, with minor modifications. ${ }^{20} 96$-well plates (Nunc, Rochester, NY, USA) were coated with the indicated concentrations of ISM or ECM proteins (Sigma, St. Louis, MO, USA) at $4^{\circ} \mathrm{C}$ overnight. Nonspecific binding sites were blocked with $1 \%$ BSA. Subconfluent ECs were harvested and suspended in CSC basal medium. Cells $\left(2 \times 10^{4}\right)$ were plated to each well and allowed to attach for $60 \mathrm{~min}$ at $37^{\circ} \mathrm{C}$ in $\mathrm{CO} 2$ incubator. In some experiments, ECs were pre-incubated for $30 \mathrm{~min}$ with integrin-specific antibodies ( $\alpha$ v antibody (clone M9), $\beta 1$ antibody (clone P4C10), $\beta 3$ antibody (clone 25E11), $\beta 5$ antibody (clone E19), $\alpha v \beta 3$ antibody (clone LM609), $\alpha \mathrm{v} \beta 5$ antibody (clone P1F6)) (Millipore, Billerica, MA, USA) or control IgG prior to their addition to the wells. After removal of the nonattached cells by washing with PBS, attached cells were fixed with 10\% formalin (Sigma) and stained with $0.2 \%$ crystal violet. The crystal violet was extracted by $10 \%$ acetic acid and cell attachment was quantified by measuring the absorbance of eluted dye at $595 \mathrm{~nm}$ with a microplate reader.
Apoptosis assays. ECs $\left(1 \times 10^{4}\right.$ cells per well) in 96-well plate were starved in basal CSC medium containing $2 \%$ FBS overnight before treated with $1 \mu \mathrm{M} \mathrm{ISM}$ and $15 \mathrm{ng} / \mathrm{ml} \mathrm{VEGF}$ for $24 \mathrm{~h}$. Apoptosis was determined by measuring cytosolic oligonucleosome-bound DNA using a Cell Death ELISA kit (Roche Diagnostics Asia Pacific, Singapore). Caspase inhibitors Z-DEVD-FMK (caspase 3 inhibitor), Z-IEDT-FMK (caspase 8 inhibitor), Z-LEHD-FMK (caspase 9 inhibitor) and Kp7-6 (Fas antagonist) (Merck, Singapore) were added to cells $1 \mathrm{~h}$ prior to treatment with ISM and VEGF.

Cell proliferation assay. ECs or siRNA transfected ECs $\left(2 \times 10^{4}\right.$ per well) were seeded in a gelatin-coated 96-well plate with CSC complete medium for $24 \mathrm{~h}$ at $37^{\circ} \mathrm{C}$ and $5 \% \mathrm{CO}$. Cells were starved with CSC basal medium supplemented with $2 \%$ FBS overnight. Then $1 \mu \mathrm{M}$ ISM and $15 \mathrm{ng} / \mathrm{ml}$ VEGF were added at the beginning of the proliferation assay. ECs were allowed to proliferate for $24 \mathrm{~h}$ and 


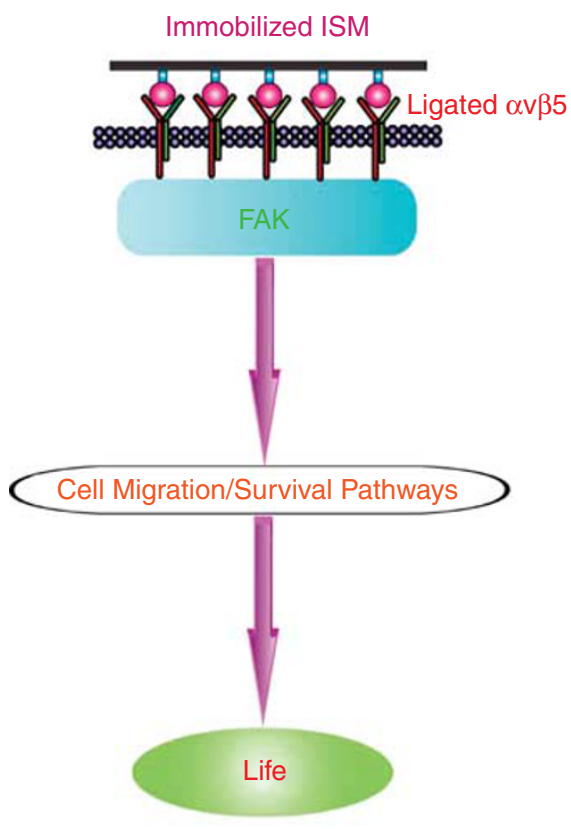

IMD

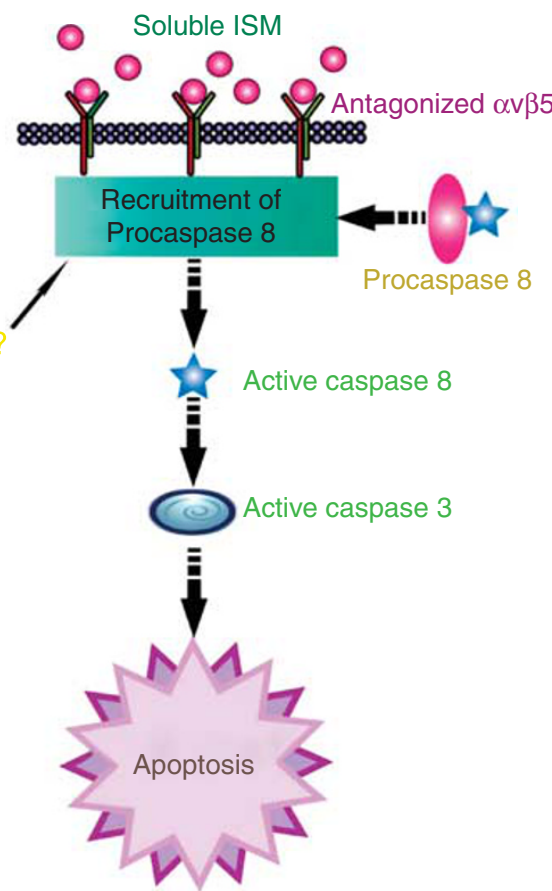

Figure 8 Schematic illustration of the dual functions of ISM. (a) Immobilized ISM serves as integrin agonist to mediate cell survival/migration by activating FAK. (b) Soluble ISM induces cell apoptosis through IMD (direct activation of caspase-8). '?' indicates the unresolved question if additional molecules are involved in the integrin-caspase-8 complex formation

proliferation was determined by BrdU labeling (Millipore), according to manufacturer's instruction.

ELISA measurement of free $\alpha \mathrm{v} \beta 5$ integrin. A total of 96 -well plates were coated with VN $(50 \mu \mathrm{g} / \mathrm{ml})$, FN $(50 \mu \mathrm{g} / \mathrm{ml})$ and ISM $(1 \mu \mathrm{M})$ at $37^{\circ} \mathrm{C}$ for $2 \mathrm{~h}$. Nonspecific binding sites were blocked with $1 \%$ BSA in PBS for $2 \mathrm{~h}$ at room temperature. HUVECs $\left(2 \times 10^{4}\right.$ cells) were seeded into each well and allowed to attach overnight. After removal of the nonattached cells by washing with PBS, ECs were fixed and blocked with $3 \% \mathrm{BSA}$ prior to incubating with anti- $\alpha \mathrm{V} \beta 5$ antibody (clone P1F76, Santa Cruz, Santa Cruz, CA, USA) for $1 \mathrm{~h}$. Detection of integrin was achieved by anti-mouse IgG-HRP and developed with TMB $\left(3,3^{\prime}, 5,5^{\prime}\right.$ tetramethylbenzidine) (Dako, Glostrup, Denmark) for $15 \mathrm{~min}$. The reaction was stopped with the addition of $50 \mu \mathrm{l} 1 \mathrm{M} \mathrm{H}_{2} \mathrm{SO}_{4}$. The absorbance was then determined at $450 \mathrm{~nm}$.

Immunofluorescence staining. ECs were plated for $2 \mathrm{~h}$ on $96-$ well plates coated with $20 \mu \mathrm{g} / \mathrm{ml}$ of either VN or ISM. Cells were rinsed with PBS twice, fixed with $4 \%$ paraphormaldehyde in PBS, permeabilized with $0.1 \%$ Triton X-100 in PBS for $10 \mathrm{~min}$ and blocked with $3 \% \mathrm{BSA}$ for $1 \mathrm{~h}$ at room temperature. Cells were stained with rhodamine-conjugated phalloidine (actin stress fibers) or goat anti- $\beta 5$ antibody (Santa Cruz) with FITC-labeled rabbit anti-goat IgG (Invitrogen, Carlsbad, CA, USA) and counter-stained with DAPI (Sigma). For ISM binding to ECs, cells were incubated with ISM for $1 \mathrm{~h}$ at $37^{\circ} \mathrm{C}$ and $5 \% \mathrm{CO}_{2}$ before they are fixed and stained with mouse anti-His tag (sc-57598, Santa Cruz) and goat anti- $\beta 5$ (sc-5401, Santa Cruz) in $3 \%$ BSA for $1 \mathrm{~h}$. ISM and $\beta 5$ integrin were then visualized with a mix of Alexa Fluor 488 rabbit anti-mouse IgG and Alexa Fluor 568 rabbit anti-goat IgG (Invitrogen). Fluorescence images were obtained with a Zeiss laser scanning confocal microscope system (Carl Zeiss South East Asia, Singapore).

RNAi mediated gene silencing. siRNAs were purchased from 1st BASE (Singapore): siRNA targeting human $\beta 3$ integrin subunit mRNA (5'-CAAGCCUG UGUCACCAUAC-dTdT-3'); human $\beta 5$ subunit integrin mRNA ( $5^{\prime}$-GCUCGCAG GUCUCAACAUA-dTdT-3'); and a random sequence control siScramble (5'-GACGUGGGACUGAAGGGGU-dTdT-3') were as described before. ${ }^{21}$
Determination of caspase activation by immunoblot. Activation of caspases 3,8 , and 9 was determined as a function of procaspase cleavage. ${ }^{22} \mathrm{ECs}$ $\left(1 \times 10^{5} / \mathrm{ml}\right)$ were incubated with ISM $(1 \mu \mathrm{M})$ and VEGF $(15 \mathrm{ng} / \mathrm{ml})$ for $24 \mathrm{~h}$. Caspase activation and PARP cleavage were analyzed at $0,2,4,8,16$ and $24 \mathrm{~h}$ post ISM-treatment. Cell lysates were separated by SDS-PAGE and probed with antibodies to caspase 3 (R\&D systems, Minneapolis, MN, USA), caspase 8 , caspase 9, PARP (Millipore).

Co-immunoprecipitation. ECs were incubated with $1 \mu \mathrm{M}$ ISM for $16 \mathrm{~h}$ at $37^{\circ} \mathrm{C}, 5 \% \mathrm{CO}$. After removing the unbound ISM, cells were washed, lysed and cell lysates were incubated with anti- $\alpha \mathrm{v} \beta 5$ antibody, control $\lg G$ and protein $A / G$ Sepharose beads (Santa Cruz) for $2 \mathrm{~h}$ at $4^{\circ} \mathrm{C}$. The precipitates were resolved and analyzed for the presence of caspase-8 or FADD by immunoblotting.

Cell haptotaxis assay. Haptotactic cell motility was measured by using $8 \mu \mathrm{m}$ cell culture inserts, as previously described. ${ }^{23}$ The undersurface of the membrane filter was pre-coated with serial concentration of BSA, ISM or FN. ECs $\left(1 \times 10^{6}\right.$ cells $/ \mathrm{ml}$ ) were added to each insert. The cells were allowed to migrate for $6 \mathrm{~h}$ at $37^{\circ} \mathrm{C}$ and $5 \% \mathrm{CO} 2$ before being fixed and stained with crystal violet. Cell migration was quantified by measuring the absorbance of eluted crystal violet at $560 \mathrm{~nm}$. Antiintegrin antibodies were used at $10 \mu \mathrm{g} / \mathrm{ml}$ in the antibody-neutralization experiments.

Determination of integrin binding site of ISM. AMOP-N and AMOP-C region were cloned into $\mathrm{PET}-\mathrm{M}$ vector (as described in reference 4) using the following primers: AMOPF1EcoRl: CCGGAATTCCGGCTGCTTGCGGG AAGTGA GGAGT; AMOPR1Xhol: CCGCTCGAGCGGCTCCAGGGACAGCATAGA GCGGA; AMOPF2ECORl: CCGGAATTCCGGTGCATCCGCTCTATGCTGTCCCT; AMOPR2Xhol: CCGCTCGAGTCGGTCTGGCTTCTTGGAACTGTTTG. The point mutants denoted AMOPKD316AA was obtained from a pET-M-AMOP plasmid using site-directed mutagenesis procedures (Stratagene, La Jolla, CA, USA). The recombinant proteins were expressed in E.coli (BL21DE3) and purified using Ni-NTA affinity chromatography in $6 \mathrm{M}$ urea, according to the manufactures' instructions (Promega, Madison, WI, USA). The proteins were then further purified by reverse-phase HPLC. Protein concentration was determined using BioRad Bradford assay reagent 
(BIORAD, Hercules, CA, USA). Cell attachment assays were then used to determine the ability of various protein fragments to support EC adhesion, as described in the above section.

Statistical analysis. All data values were reported as mean \pm S.E.M. Statistical differences were determined using the Student's $t$-test. $P$-values of 0.05 were considered to be statistically significant.

\section{Conflict of Interest}

The authors declare no conflict of interest.

Acknowledgements. This work is supported by a grant from the Singapore Biomedical Research Council (BMRC07/1/21/19/493) to Ruowen Ge.

1. Carmeliet P. Angiogenesis in life, disease and medicine. Nature 2005; 438: 932-936.

2. Ribatti $D$. The discovery of antiangiogenic molecules: a historical review. Curr Pharm Des 2009; 15: 345-352.

3. Pera EM, Kim JI, Martinez SL, Brechner M, Li SY, Wessely $O$ et al. Isthmin is a novel secreted protein expressed as part of the Fgf- 8 synexpression group in the Xenopus midbrain-hindbrain organizer. Mech Dev 2002; 116: 169-172.

4. Xiang W, Ke Z, Zhang Y, Cheng GH, Irwan ID, Sulochana KN et al. Isthmin is a novel secreted angiogenesis inhibitor that inhibits tumor growth in mice. J Cell Mol Med 2011; 15: 359-374.

5. Silva R, D'Amico G, Hodivala-Dilke KM, Reynolds LE. Integrins: the keys to unlocking angiogenesis. Arterioscler Thromb Vasc Biol 2008; 28: 1703-1713.

6. Alghisi GC, Ponsonnet L, Ruegg $\mathrm{C}$. The integrin antagonist cilengitide activates alphaVbeta3, disrupts VE-cadherin localization at cell junctions and enhances permeability in endothelial cells. PLoS One 2009; 4: e4449.

7. Yamamoto M, Yamato M, Aoyagi M, Yamamoto K. Identification of integrins involved in cell adhesion to native and denatured type I collagens and the phenotypic transition of rabbit arterial smooth muscle cells. Exp Cell Res 1995; 219: 249-256.

8. Eskens FA, Dumez H, Hoekstra R, Perschl A, Brindley C, Bottcher S et al. Phase I and pharmacokinetic study of continuous twice weekly intravenous administration of Cilengitide (EMD 121974), a novel inhibitor of the integrins alphavbeta3 and alphavbeta5 in patients with advanced solid tumours. Eur J Cancer 2003; 39: 917-926.

9. Maubant S, Saint-Dizier D, Boutillon M, Perron-Sierra F, Casara PJ, Hickman JA et al. Blockade of alpha $v$ beta3 and alpha $v$ beta5 integrins by RGD mimetics induces anoikis and not integrin-mediated death in human endothelial cells. Blood 2006; 108: 3035-3044.
10. Stupack DG, Puente XS, Boutsaboualoy S, Storgard CM, Cheresh DA. Apoptosis of adherent cells by recruitment of caspase-8 to unligated integrins. J Cell Biol 2001; 155: 459-470.

11. Schwartz MA, Schaller MD, Ginsberg MH. Integrins: emerging paradigms of signal transduction. Annu Rev Cell Dev Biol 1995; 11: 549-599.

12. Ruegg C, Yilmaz A, Bieler G, Bamat J, Chaubert P, Lejeune FJ. Evidence for the involvement of endothelial cell integrin alphaVbeta3 in the disruption of the tumor vasculature induced by TNF and IFN-gamma. Nat Med 1998; 4: 408-414.

13. Golubovskaya VM, Kweh FA, Cance WG. Focal adhesion kinase and cancer. Histol Histopathol 2009; 24: 503-510.

14. Burridge K, Turner CE, Romer LH. Tyrosine phosphorylation of paxillin and pp125FAK accompanies cell adhesion to extracellular matrix: a role in cytoskeletal assembly. $J$ Cell Biol 1992; 119: 893-903.

15. Sospedra P, Mestres C, Haro I, Munoz M, Busquets MA. Effect of amino acid sequence change on peptide-membrane interaction. Langmuir 2002; 18: 1231-1237.

16. Brassard DL, Maxwell E, Malkowski M, Nagabhushan TL, Kumar CC, Armstrong L. Integrin alpha(v)beta(3)-mediated activation of apoptosis. Exp Cell Res 1999; 251: 33-45.

17. Stupack DG, Cheresh DA. Apoptotic cues from the extracellular matrix: regulators of angiogenesis. Oncogene 2003; 22: 9022-9029.

18. Rehn $M$, Veikkola $T$, Kukk-Valdre $E$, Nakamura $H$, Ilmonen $M$, Lombardo $C$ et al Interaction of endostatin with integrins implicated in angiogenesis. Proc Natl Acad Sci USA 2001; 98: 1024-1029.

19. Stupack DG. Integrins as a distinct subtype of dependence receptors. Cell Death Differ 2005; 12: 1021-1030.

20. Eikesdal HP, Sugimoto H, Birrane G, Maeshima Y, Cooke VG, Kieran M et al. Identification of amino acids essential for the antiangiogenic activity of tumstatin and its use in combination antitumor activity. Proc Natl Acad Sci USA 2008; 105: 15040-15045.

21. Monferran S, Skuli N, Delmas C, Favre G, Bonnet J, Cohen-Jonathan-Moyal E et al. Alphavbeta3 and alphavbeta5 integrins control glioma cell response to ionising radiation through ILK and RhoB. Int J Cancer 2008; 123: 357-364.

22. Buckley CD, Pilling D, Henriquez NV, Parsonage G, Threlfall K, Scheel-Toellner D et al. RGD peptides induce apoptosis by direct caspase-3 activation. Nature 1999; 397: 534-539.

23. McCarthy JB, Furcht LT. Laminin and fibronectin promote the haptotactic migration of B16 mouse melanoma cells in vitro. J Cell Biol 1984; 98: 1474-1480.

Cell Death and Disease is an open-access journal published by Nature Publishing Group. This work is licensed under the Creative Commons Attribution-Noncommercial-No Derivative Works 3.0 Unported License. To view a copy of this license, visit http://creativecommons.org/licenses/by-nc-nd/3.0/

\section{Supplementary Information accompanies the paper on Cell Death and Disease website (http://www.nature.com/cddis)}

